\title{
AERODYNAMIC CHARACTERISTICS OF THE X-TAIL STABILIZER IN A HYBRID UNMANNED AIRCRAFT
}

\author{
Czyz, Z.* \& Karpinski, P.** \\ * Aeronautics Faculty, Military University of Aviation, 35 Dywizjonu 303 St., 08-521 Dęblin, Poland \\ ** Department of Thermodynamics, Fluid Mechanics and Aviation Propulsion Systems, \\ Faculty of Mechanical Engineering, Lublin University of Technology, 36 Nadbystrzycka St., \\ 20-618 Lublin, Poland \\ E-Mail: z.czyz@law.mil.pl, pawel.karpinski@ pollub.edu.pl
}

\begin{abstract}
The paper presents the results of the numerical calculation of the flow around hybrid unmanned aircraft with X-tail stabilizers. This aircraft design is an innovative combination of a gyroplane and a multirotor. The aerodynamic properties of the two-part X-tail stabilizer were analysed. The aerodynamic forces and moments on this element of the aircraft were specified from the CFD simulation calculations for the defined values of the angle of attack and the slip angle, and particular attention was paid to the differences between the upper and lower parts of the stabilizer. In addition, the turbulence kinetic energy on the surface of the vortex core region were analysed to conduct a qualitative and quantitative analysis of energy losses in flow, resulting from local separation and vortices. The obtained results allow for conducting further aerodynamic comparative analyses on other types of stabilizers.

(Received in August 2020, accepted in October 2020. This paper was with the authors 3 weeks for 2 revisions.)
\end{abstract}

Key Words: Aerodynamic Characteristics, Autogyro, Hybrid Aircraft, Multicopter, Stabilizer

\section{INTRODUCTION}

The most popular methods of aerodynamic research include experimental wind tunnel tests and CFD (Computational Fluid Dynamics) simulation calculations. An important advantage of the latter is the speed of obtaining results and the ability to analyse many research configurations, which significantly reduces the cost of research. There are many works devoted to the use of this methodology for testing aerodynamics of aircraft and their components [1-4]. This method can also be used to analyse fluid flow in various mechanical devices, e.g., nozzle for a pulse jet system [5]. Experimental wind tunnel research, in turn, can be a source of appropriately accurate and detailed aerodynamic database for further modelling. [6] The determined coefficients then form the basis for validation of computer models.

In recent years, interest in gyroplanes has increased in the civil aviation. The light and simple design and low operating costs mean that these aircraft are often used for transport and recreational flights. In addition, new variants of aircraft designs based on gyroplanes are created, such as: a convertiplane (an autogyro with wings) [7], a hybrid drone combining the features of a gyroplane and a helicopter [8] or a hybrid combination of a gyroplane and a multirotor being the subject of this study so this type of rotorcraft should be investigated in terms of its aerodynamic properties and stability characteristics to ensure safety [9]. An example of such research is the work [10] that presents an experimental gyroplane study including aerodynamic characteristics, the pitch moment coefficient in relationship to an angle of attack for various gyroplane configurations (with and without a tail). The basic aerodynamic properties of a gyroplane in a wind tunnel are researched in the paper [11]. Another example is the work $[12,13]$ in which the Particle Image Velocimetry (PIV) method is used to visualize the velocity field around the gyroplane.

This work is part of a series of publications devoted to the numerical analysis of aerodynamics of light rotorcraft. Aerodynamic characteristics of gyroplanes have been studied 
in the previous works using the CFD method. The study [14] presents tests of aerodynamic forces and moments generated on individual autogyro elements. It has been shown that for large values of the angle of attack, the stabilizer can be a source not only of a high pitch moment, but also of a high drag force comparable to the resistance of the fuselage. The effect of a sideslip angle on load of the gyrocopter stabilizer with an inverted twin tail is examined in the work [15] and aerodynamic forces acting on vertical and horizontal stabilizers as well as pressure distribution on their surfaces are obtained. Gyroplane longitudinal static stability for the selected stabilizer angles was also studied [16]. The pitch moment, which determines the longitudinal stability of the aircraft, is particularly important. It has been shown that the angle of the stabilizer relative to airflow has the greatest impact on the stability of the tested aircraft for the extreme negative angle of attack. The issue of gyroplane stability is also researched in [17-20].

A separate issue in aircraft aerodynamics studies is stabilizer aerodynamics in rotorcraft. Stabilizers used in gyroplanes and rotor hybrid aircraft are structurally different from those used in helicopters because a tail boom in classic helicopters has a tail rotor that affects the distribution of forces and aerodynamic moments of the entire structure. The theory devoted to the issue of horizontal and vertical stabilizers in helicopters is presented in [21]. The paper [22] analyses the aerodynamic design of the helicopter horizontal stabilizer. This study shows that the results obtained from flight tests and simulation analysis give an accurate estimation of helicopter longitudinal behaviour. An interesting numerical research into a helicopter stabilizer including rotor downwash in forward-flight is presented in [23]. The influence of geometrical modifications in stabilizer planform and sections and the angle of its setting on stability and control characteristics of the empennage are investigated. The work [24], in turn, presents the research on the influence of a gyroplane rotor on the aerodynamics of the stabilizer. The CFD analysis shows that the most important aerodynamic coefficients and stability derivatives of the gyroplane body can change because of rotor operation. Airflow around the helicopter, including the tail boom with the stabilizer is investigated in an experimental study combined with some numerical tests [25]. Similar numerical tests of aerodynamic forces on the helicopter fuselage depending on the angle of attack and the yaw angle are presented in [26]. The determined aerodynamic forces and moments on individual aircraft components can then be used to calculate aerodynamic coefficients, useful in comparative aerodynamic performance analyses. These coefficients can also be determined by modelling [27].

For gyroplanes, the stabilizer is often vertical or horizontal aerofoil-shaped elements mounted on a single or double tail boom. These elements can be arranged in various configurations, e.g., one long horizontal stabilizer with two vertical stabilizers or a double or triple vertical stabilizer on a horizontal beam. This work focuses on the analysis of the aerodynamic characteristics of the X-tail stabilizer which is a combination of a gyroplane and a multi-rotor, mounted in hybrid unmanned aircraft. The use of such a stabilizer makes it possible to install engine nacelles for driving additional propellers with an adjustable thrust vector and elements fastening the rear wheels to the aircraft. However, it is necessary to assess the aerodynamic properties of this type of stabilizer in terms of generated aerodynamic forces and moments in different flight conditions (different angle of attack and sideslip angle) in a steady state.

The article analyses the new design of the aircraft with a new type of stabilizer. The stabilizer was designed by the authors of the work. During the design work, stabilizer shapes used so far in aviation were used, mainly conventional and inverted V-tail. The proposed X-tail solution is a variation of both of these types of tail in a single boom arrangement. This approach represents a new, original concept of stabilizer geometry that the authors planned to investigate in a simulation. The original contribution is an analysis of forces and aerodynamic moments, as well as a vortex core region for a new type X-tail stabilizer. The authors of the study were 
interested in the share of the upper and lower part of the stabilizer in the generated forces and aerodynamic moments. The longitudinal stability of the proposed solution was also analysed.

\section{RESEARCH OBJECT AND SCOPE OF RESEARCH}

The research object was a hybrid unmanned aerial vehicle combining a gyroplane and a multirotor (see Fig. 1). The combination of two different propulsion systems improves operational capabilities of the aircraft and its safety. The core of the design consists of a fuselage with an autorotational main rotor as in a classic gyroplane, which gives a light and simple structure. Additional horizontally arranged rotors allow for hovering and vertical take-off and landing, which works also as a redundancy of the propulsion system so even if the main rotor fails, safe landing is still possible. The presented aircraft model (see Fig. 1) is a developmental concept at design and numerical research stages. The aircraft will be ultimately equipped with a main rotor with a diameter not exceeding $75^{\prime \prime}(1905 \mathrm{~mm})$ and four additional rotors, each with a diameter not exceeding 13" (330.2 mm), arranged symmetrically around the fuselage. Fig. 1 shows a simplified model of the aircraft with an X-tail stabilizer which was subjected to aerodynamic analysis. Rear wheels will eventually be attached to the bottom edges of the lower stabilizer. Rear engine nacelles are attached at the ends of the upper stabilizer. The upper stabilizer aerofoils are slightly bended at a distance from the tail beam. The figure shows the right-handed Cartesian coordinate system and the directions of measuring the angle of attack and the slip angle. Both angles are considered positive when the direction of rotation is clockwise. The geometric dimensions of the aircraft model are: height $467 \mathrm{~mm}$, length $1042 \mathrm{~mm}$, width $562 \mathrm{~mm}$.
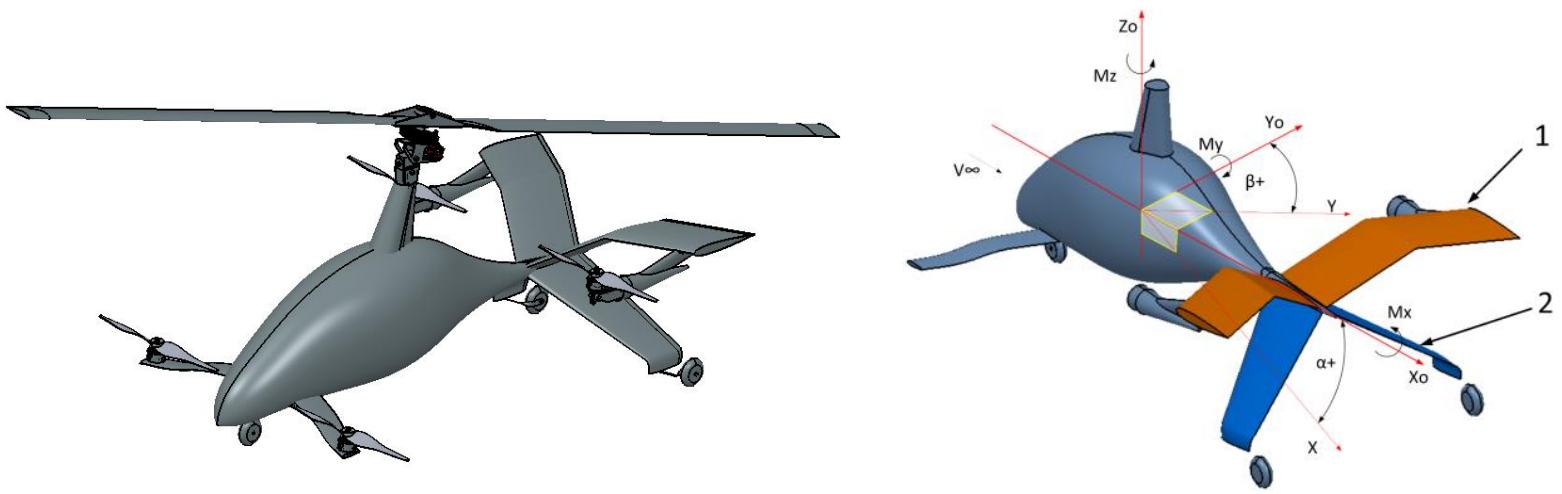

Figure 1: General view of the research object (left) and aircraft model (right) with highlighted stabilizers and the coordinate system: 1 - upper stabilizer, 2 - lower stabilizer.

The geometric model of the aircraft was developed in the Catia V5 software and simplified for this CFD analysis by subtracting its rotors, motors and fastening elements. However, other elements such as its fuselage, arms or rear engine nacelles were taken into account while affecting the airflow around the tested stabilizer. The developed geometry was introduced to the Design Modeler module in the Ansys Workbench software. Next, the angle of attack and the sideslip angle were parameterized for each of the considered configurations. The test object was placed in the geometric centre of the cuboidal computational domain. Its dimensions are $4562 \times 7042 \times 4467 \mathrm{~mm}$, height, length and width, respectively. A velocity based inlet and a pressure based outlet are defined on its surfaces. The surface of the study object was defined as a wall in individual sections.

In the Mesh module, a computational grid consisting of tetrahedron-type elements was generated on the surface of the aircraft model. The object was divided into several components to read aerodynamic forces and moments occurring on their surfaces. In this work, special attention was paid to the stabilizer, so the mesh density on this element was increased. In addition, the mesh density at the boundary layer was increased for the entire research object 
using the Inflation function with the Smooth Transition function and the parameters like Transition Ratio 0.272, Maximum Layers 5 and Growth Rate 1.2. Fig. 2 shows the generated mesh of 3,450,608 elements for the entire aircraft and its stabilizers. The number of mesh elements and their size have been selected so that further enlargement of the grid generates differences in results not exceeding $1 \%$. For the entire research object, the value of $y+$ is in the range from 0.73 to 56.06 . Considering the stabilizer itself, the maximum value is reduced to 42.93. The use of wall functions is therefore justified.

Due to the low velocity of the air inside the computational domain, pressure-based calculation was adopted. The object was analysed for steady-state flow. For the turbulence model, the $k-\omega$ SST was selected since it offered a faster convergence and better results compared to $k-\varepsilon$. It enabled to use the $k-\varepsilon$ model in the outer region and outside of the boundary layer and the $k-\omega$ in the inner boundary layer.
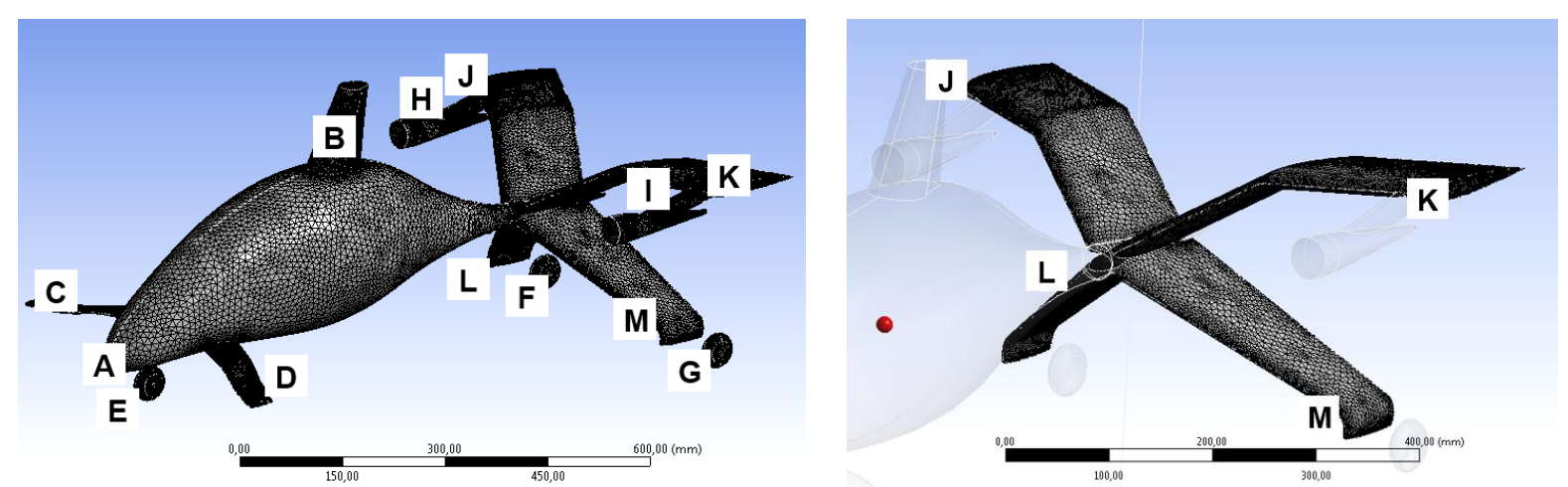

Figure 2: General view of the generated mesh on the aircraft and stabilizer: A - fuselage, B - mast, C right front arm, D - left front arm, E - front wheel, F - right rear wheel, G - left rear wheel, $\mathrm{H}$ - right engine nacelle, $\mathrm{I}$ - left engine nacelle, $\mathrm{J}$ - upper right stabilizer, $\mathrm{K}$ - upper left stabilizer, $\mathrm{L}$ - lower right stabilizer, $\mathrm{M}$ - lower left stabilizer.

The model takes into account the kinetic energy of turbulence and specific dissipation of kinetic energy. The $k-\omega$ equation finds application in flow calculations for inner parts of the boundary layer. The shear stress transport (SST) formulation enables a smooth transition to $k-\varepsilon$ in a free stream so a high sensitivity of the model to inlet free-stream turbulence properties is avoided. In addition, the model provides faithful results for large pressure gradients and flow separation. The assumed level of turbulence intensity is $1 \%$ and the turbulence length scale is $0.28 \mathrm{~m}$ on the inlet and outlet surface. The working gas was assumed to be air defined as ideal gas. The calculations were carried out for the flow velocity of $20 \mathrm{~m} / \mathrm{s}$ defined on the inlet surface. The air density corresponded to normal conditions, i.e., temperature of $15{ }^{\circ} \mathrm{C}$ and pressure of $1013.25 \mathrm{hPa}$. Its dynamic viscosity was set to $17.894 \mu \mathrm{Pa} \cdot \mathrm{s}$.

For the created computational model, a series of simulations was carried out for the assumed configurations of the attack angle and the sideslip angle. The angle of attack was changed in the range from $-20^{\circ}$ to $+20^{\circ}$, every $5^{\circ}$, and the sideslip angle changed in the range from $0^{\circ}$ to $+20^{\circ}$, every $5^{\circ}$.

\section{RESULTS AND ANALYSIS}

The following figures show the aerodynamic forces and moments calculated for the selected configurations of the angle of attack and the sideslip angle for the upper and lower stabilizers. These relationships are aimed at conducting quantitative analysis and assessing the percentage share of individual parts of the entire stabilizer in generating forces and moments. Due to the large amount of generated data and in order to simplify the analysis, it was decided to present these parameters for two extreme sideslip angles, i.e., $\beta=0^{\circ}$ and $\beta=20^{\circ}$. 
Analysing the drag force (see Fig. 3), it can be concluded that the upper stabilizer generated a greater force over the entire range of angle of attack than the lower stabilizer. The increase in the sideslip angle resulted in an increase in the drag force for all considered angles of attack for both the upper and lower stabilizers. It can also be seen that a greater force was generated for the zero sideslip angle on the upper stabilizer for the extreme angle of attack. The increase in the sideslip angle resulted in the generation of the greater drag force for the small values of angle of attack. The largest difference in the drag force on the upper and lower stabilizers was $2.9 \mathrm{~N}$ for $\alpha=-20^{\circ}$ and $\beta=0^{\circ}$. The lateral force on the upper and lower stabilizers for the selected sideslip angles is shown in Fig. 3.
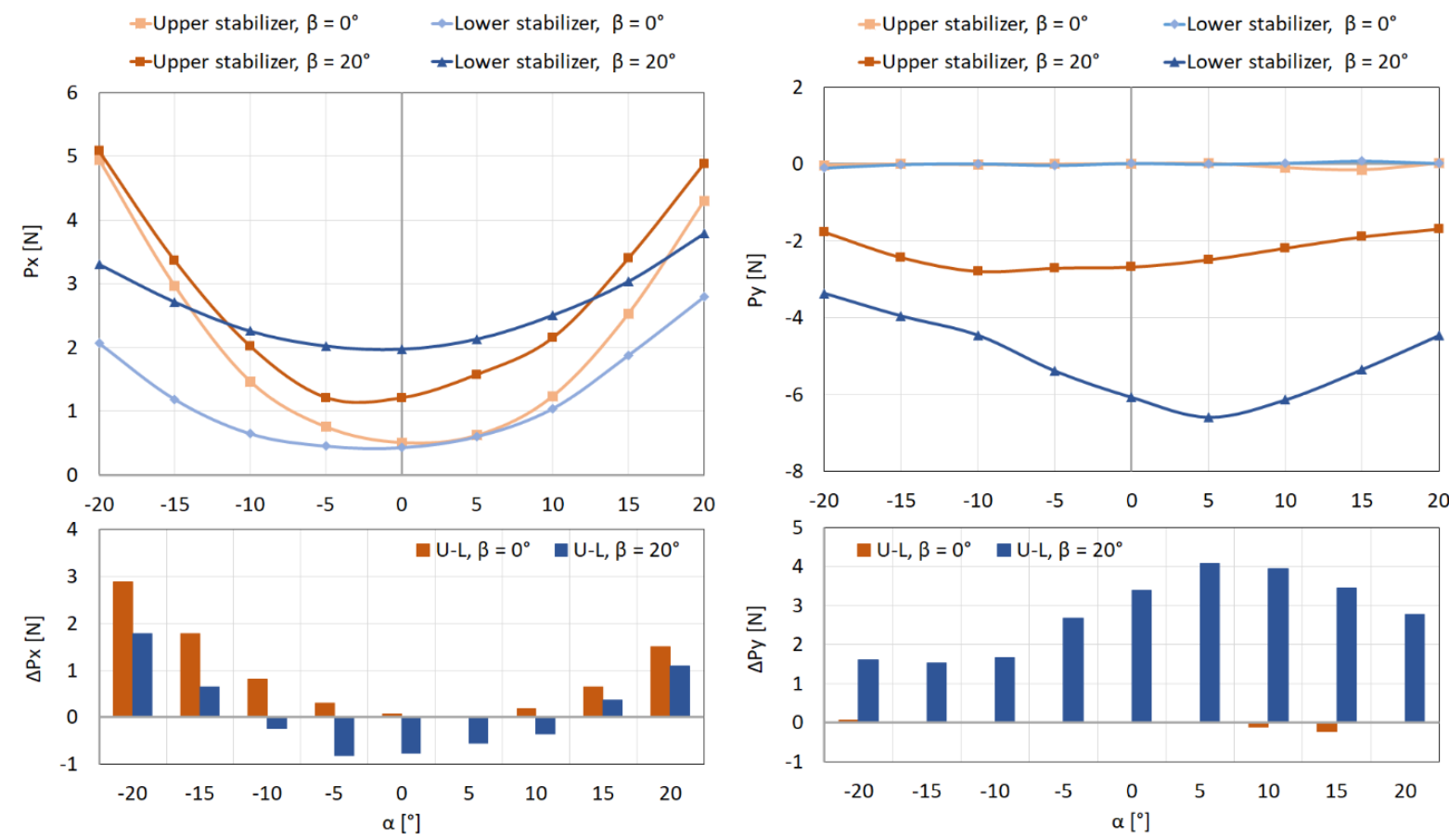

Figure 3: Comparison of the drag force (left) and lateral force (right) for upper and lower stabilizer versus the angle of attack for the selected sideslip angles.

For the zero sideslip angle, this force had a small value close to zero in the entire range of angle of attack for both the upper and lower stabilizers. An increase in the sideslip angle increased the difference between the upper and lower stabilizers. For $\beta=20^{\circ}$, the lateral force generated on the lower stabilizer was about 3 times greater than the force on the upper one. In this case, for the lower stabilizer, an extreme was observed for $\alpha=5^{\circ}$, while for the upper one there was no such extreme and the curve is flatter.

A significant change in the lift force was also observed on the individual parts of the stabilizer depending on the angle of attack and the sideslip angle (see Fig. 4). For the zero sideslip angle for the large negative angles of attack, the absolute value of force on the upper stabilizer was even twice as high as on the lower one. The highest force on the upper stabilizer was $14.7 \mathrm{~N}$ for $\alpha=20^{\circ}$, while its smallest value equal to $-15.8 \mathrm{~N}$ occurred for $\alpha=-20^{\circ}$. The transition into the positive angles of attack resulted in a change in the value sign although this trend maintained. For the large sideslip angle $\left(\beta=20^{\circ}\right)$ and negative angles of attack, the differences between the upper and lower stabilizers were smaller (except for the angle $\alpha=-5^{\circ}$ ). For the positive angles of attack for the considered sideslip angle, a greater lift force was generated at the upper stabilizer. The differences between the upper and lower stabilizers for $\beta=0^{\circ}$ and $\beta=20^{\circ}$ increased with the decrease of the angle of attack and decreased with its increase. 
The sideslip angle also had a significant effect on the roll moment (see Fig. 4). For the zero value of the sideslip angle, the roll moment was approximately zero. The increase in the sideslip angle generated a negative moment on the bottom stabilizer in the whole range of attack angles considered. The smallest moment value on this part for $\beta=20^{\circ}$ was $-1.4 \mathrm{Nm}$. On the upper stabilizer, the moment increased with increasing the angle of attack until its maximum value was reached. For $\beta=20^{\circ}$, its value was $2.3 \mathrm{Nm}$.
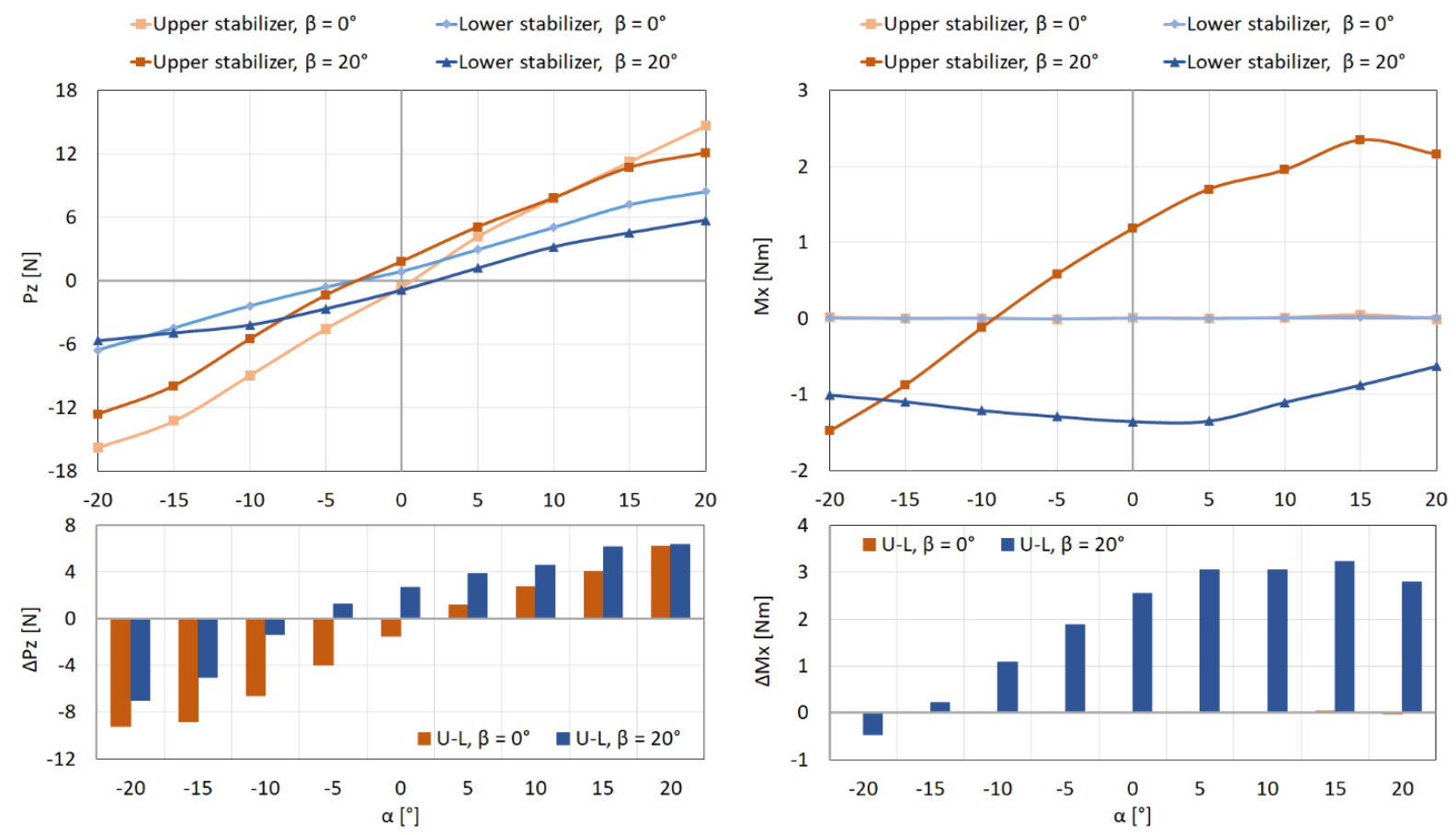

Figure 4: Comparison of the lift force (left) and roll moment (right) for the upper and lower stabilizers versus the angle of attack for the selected sideslip angles.

It was observed that for the positive values of angle of attack, an increase in the sideslip angle resulted in the changed shape of the curve for the upper stabilizer from flatter to parabolic. The angle of attack at which there was the zero roll moment on the upper stabilizer shifted towards smaller values with the increase of the sideslip angle. The moment on the upper stabilizer was greater than the moment on the lower one with the exception of the angle of attack $\alpha=-20^{\circ}$.

An inverted linear trend as for the lift force was observed for the pitch moment (see Fig. 5). For the zero sideslip angle and negative values of angle of attack, the pitch moment on the upper stabilizer was the highest. The maximum occurred for the angle of attack $\alpha=-20^{\circ}$ and was equal to $7.8 \mathrm{Nm}$. In almost the whole range of positive angles of attack, the moment on the upper stabilizer was negative. A similar trend was observed on the lower part, however, the moment values were lower than on the upper stabilizer. The increase in the sideslip angle resulted in a change in the curves slope on the graph, which translated into a decrease in both the positive and negative moments. In addition, in the range of small values of angle of attack, the absolute value of the roll moment on the upper stabilizer was greater than on the lower one. This tendency reversed the earlier, the larger the sideslip angle was. For $\beta=0^{\circ}$, the change occurred for $\alpha=5^{\circ}$, while for $\beta=20^{\circ}$ it was for $\alpha=15^{\circ}$.

The last value analysed was the yaw moment (see Fig. 5). For the zero sideslip angle, its value was approximately zero for the whole range of angle of attack. An increase in the sideslip angle resulted in a negative moment on the upper and lower stabilizers for all considered values of angle of attack. In addition, for this range, the moment on the lower stabilizer had a higher absolute value. The differences in its values increased as the angle of attack approached zero. 

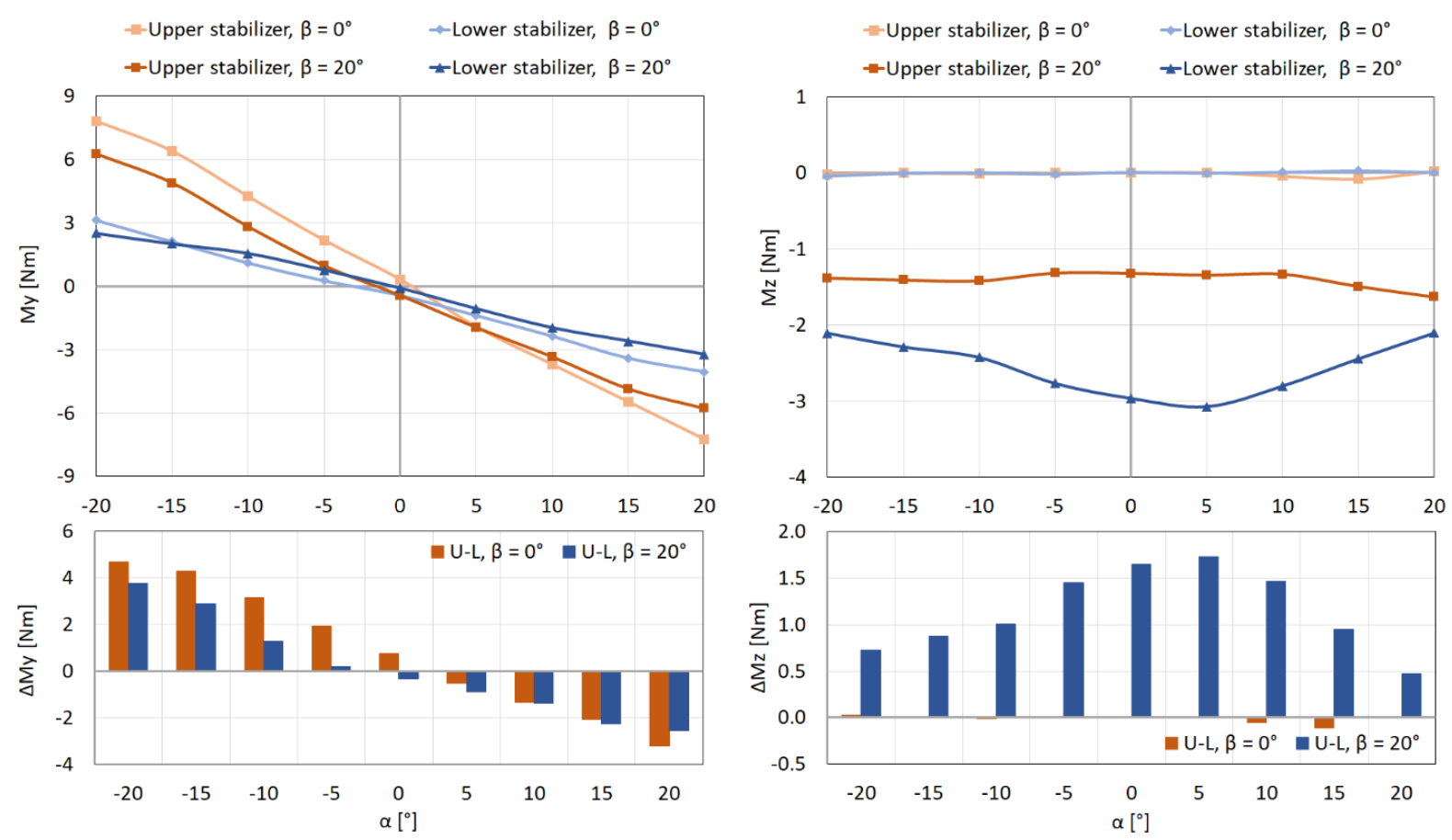

Figure 5: Comparison of the pitch moment (left) and yaw moment (right) for the upper and lower stabilizers versus the angle of attack for the selected sideslip angles.

The moment on the lower stabilizer had a relatively flat characteristics as a function of the angle of attack and for $\beta=20^{\circ}$ oscillated in the range from -1.6 to $-1.3 \mathrm{Nm}$. For the lower stabilizer, however, a clear minimum of the moment reached $-3 \mathrm{Nm}$ for $\alpha=5^{\circ}$ and $\beta=20^{\circ}$. It should be emphasized that the tendency to occur the extreme decreased with the decrease of the sideslip angle.

The formation of vortices induced behind individual structural elements like wings or a stabilizer is important for external airflow around an aircraft. A vortex should be understood as the rotational motion of many fluid particles around a common point (centre). Several papers discussed how to identify and detect the vortex core [28-30]. These methods are based on special mathematical algorithms to best represent vortex structures in the flow field obtained from numerical calculations. The work [31] is a review of such algorithms which include, among others: Helicity Method, Swirl Parameter Method, Lambda2 Method, Eigenvector Method, Maximum Vorticity Method.

With Ansys Fluent, it is possible to use one of eight different vortex detection algorithms. The program visualizes vortices as a circular or spiral set of streamlines. In this work, it was decided to use the Real Eigen Helicity method based on eigenvectors. The eigenvector method was first proposed in [32]. In the adopted method, Real Eigen Helicity is the dot product of vorticity and swirling vector that is the real eigenvector of velocity gradient tensor [33]:

$$
h=u \cdot v_{c r}
$$

where:

$h$ - real eigen helicity,

$u$ - velocity vector,

$v_{c r}-$ real eigenvector of $D$ tensor,

$D$ - velocity gradient tensor.

In general, eigenvalues of the gradient tensor $D$ satisfy the equation:

$$
\lambda^{3}+P \lambda^{2}+Q \lambda+R=0
$$

where $P, Q$ and $R$ are functions of the components of the velocity gradient tensor. 
The considered tensor has one real eigenvalue and a pair of conjugated complex eigenvalues.

For a real eigenvector, the following equation is true:

$$
\left[D-\lambda_{r} I\right] v_{r}=0
$$

where:

$\lambda_{r}$ - real eigen helicity,

$I$ - identity matrix,

$v_{r}$ - real eigenvector.

Using non-zero vectors, it is possible to calculate the real part of the complex eigenvector that satisfies the equation:

where:

$$
\left[D-\lambda_{c r} I\right] v_{c r}=-\lambda_{c i} v_{c r}
$$

$\lambda_{c r}$ - real part of the complex eigenvalue,

$v_{c r}$ - real part of the complex eigenvector.

If there are no critical points within its centre for swirling flow, velocity vectors are projected onto the plane normal to the eigenvector of the real eigenvalue to check if their values are equal to zero. If their values are equal to zero, then the point must be part of a vortex core. At the same time, it is assumed that the other two eigenvalues are complex conjugate pairs [31].

The eigenvector method was used, among others, in [34] to visualize the vortex rope, and in [35] to present vortex cores for vector fields in the transient F/A-18 simulation. This method can be used to precisely and reliably determine vortex cores in external flows [36].

Fig. 6 shows the vortex core region surface obtained by the Real Eigen Helicity method, with applied a turbulence kinetic energy $(T K E)$ distribution for the entire aircraft for the selected configurations of the angle of attack and the sideslip angle. Due to the subject of this work, the focus was on the analysis of the area around the stabilizer, comparing the values of the tested parameter around the remaining parts of the aircraft. The analysed parameter should be interpreted as energy that is accumulated in the turbulent (vortical) flow. A higher value of the tested parameter means greater energy loss resulting from turbulence and the separation of the airflow from the aerofoil surfaces and the surface of the fuselage. The vortex core region, in turn, is an iso-surface that allows a detection of vortices in a complex (multidimensional) flow field.

For the zero sideslip angle and large negative angle of attack, the highest observed value of turbulence kinetic energy was $26 \mathrm{~J} / \mathrm{kg}$. The extreme values of the tested parameter reaching $22 \mathrm{~J} / \mathrm{kg}$ appeared at the ends of the lower surface of the upper stabilizer. The zero values of angle of attack and sideslip angle decrease the maximum $T K E$ value for the entire aircraft to $18 \mathrm{~J} / \mathrm{kg}$. No deformation resulting from the vortex core region was observed on the upper and lower stabilizers, which indicates low airflow turbulence in this area.

A large positive angle of attack, in turn, caused the appearance of a strong vortex area on the surface of the stabilizers. In this case, the maximum value of turbulence energy was $28 \mathrm{~J} / \mathrm{kg}$. It is worth noting that the source of a strong flow field disturbance and occurrence of vorticity in this configuration were the engine nacelles on which $T K E$ reached its extreme values. In all described cases, strong vorticity was also observed behind the mast and arms located in the front of the fuselage.

An increase in the sideslip angle resulted in the occurrence of flow asymmetry, and thus asymmetry in the analysed kinetic energy losses. For the extreme negative angle of attack, a strong vortical area was observed on the lower surface of the lower right and upper left stabilizers. The extreme TKE value for the entire aircraft was $29 \mathrm{~J} / \mathrm{kg}$ and was higher by about $12 \%$ compared to the zero-sideslip angle configuration. 

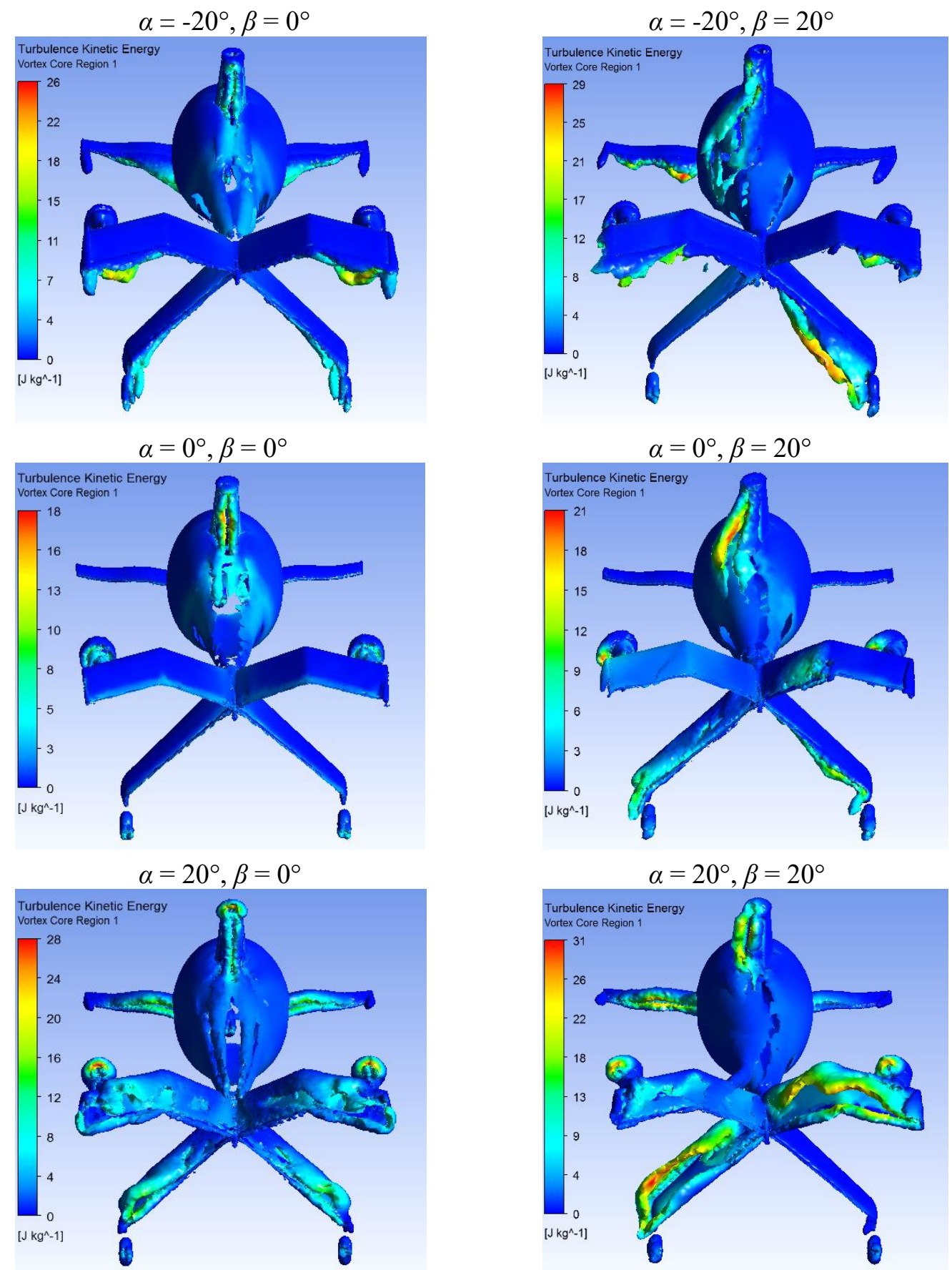

Figure 6. Vortex core region surface with the turbulence kinetic energy (TKE) distribution applied to the entire aircraft for the selected configurations of the angle of attack and the sideslip angle.

Intense turbulence also occurred on the left arm and left side of the fuselage because of the separation of incoming airflow on this part of the aircraft. In the case of the zero sideslip angle, the vorticity on both the stabilizer and the entire aircraft decreased. Turbulence kinetic energy reached a maximum value of $21 \mathrm{~J} / \mathrm{kg}$, which was also a higher value compared to the zero sideslip angle. The increased TKE value on the vortex core region surface occurred on the upper surface of the lower left and upper right stabilizers and on the lower surface of the right lower stabilizer. In the case of a large positive angle of attack and a large sideslip angle $\left(\beta=20^{\circ}\right)$, the highest TKE value $(31 \mathrm{~J} / \mathrm{kg})$ occurred in all cases considered. A large deviation of the aircraft from the reference position results in a strong vorticity on the upper surface of the upper stabilizer, especially its right part, and the upper surface of the left lower stabilizer. On the latter 
surface, there was an extreme value of turbulence kinetic energy, especially around the leading edge. For a large sideslip angle, strong vorticity arises behind the mast and arm from the incoming airflow. Regardless of the sideslip angle, a large positive angle of attack is a source of vorticity around both engine nacelles.

\section{CONCLUSION}

The study examined the X-tail stabilizer in a hybrid aircraft being a combination of a gyroplane and a multi-rotor. The calculations specified aerodynamic forces and moments on the analysed elements.

The obtained values of aerodynamic forces changed significantly depending on the sideslip angle and angle of attack. In general, an increase in the sideslip angle results in an increase in the drag force and lateral force on the stabilizer throughout the considered range of angle of attack. The lift force, in turn, decreases as the sideslip angle increases. The sideslip angle also significantly affects aerodynamic moments. With its increase, the roll moment increases and the pitch moment and yaw moment decrease.

The large sideslip angle also causes significant differences in aerodynamic forces and moments on the upper and lower stabilizers. In addition, these differences change as the angle of attack increases. It is the lower stabilizer that plays the dominant role in the lateral force, roll moment and yaw moment. For the negative values of angle of attack and zero sideslip angles, the upper stabilizer prevails in lift and pitch. For the positive angles, the lift force is greater at the bottom stabilizer, while in the case of pitch, the lower stabilizer generally dominates (except for the extreme $\alpha$ value). For the drag force, the upper stabilizer is especially responsible for the extreme values of the angle of attack, while for its small values the lower stabilizer generates greater forces.

An increase in the sideslip angle results in an increase in the maximum value of kinetic energy on the surface of the vortex core region. For the angle $\beta=20^{\circ}$, the increase in energy average several percent. The highest value of turbulence kinetic energy occurs for $\alpha=20^{\circ}$ and $\beta=20^{\circ}$. This is due to the fact that with a large deviation of the aircraft from the reference position, the flow over the surface of the aircraft fuselage and stabilizer is not laminar. There are flow separation and local turbulence, especially on the left side of the fuselage, the upper surface of the upper right and lower left stabilizers. For a large negative angle of attack, there is symmetrical turbulence on the upper surface of the upper left and lower right stabilizers. For the zero sideslip angle and a large positive angle of attack, a strong vorticity is observed behind the trailing edges of all stabilizer parts.

For the considered parts of the stabilizer and the defined values of the beta angle, a decreasing linear trend of pitching moment was observed with the increase of the angle of attack. That means that the entire stabilizer features good longitudinal stability. This is due to the high lift generated by the stabilizer (especially the upper one). Yawing moment maintains a relatively low variability as a function of angle of attack and takes negative values over the entire range. Maintaining this kind of stability is crucial at high values of angle of attack to avoid phenomena such as stalls and spins when manoeuvring. For $\beta=0^{\circ}$, the upper stabilizer generated greater drag force compared to the lower one over the entire range of angle of attack. The lower stabilizer played the dominant role for a high sideslip angle in the range of small values of angle of attack $\left(-11^{\circ}\right.$ to $\left.+13^{\circ}\right)$. This is the effect of additional horizontal surfaces that increase drag force, especially at extreme values of angle of attack. The use of the proposed stabilizer concept for the research object is justified as it plays the role of both the support structure (arms) for the engine nacelles and the undercarriage. In addition, the lower part of the stabilizer significantly contributes to the generation of the pitching moment. For the smallest considered angle of attack, i.e. $-20^{\circ}$, this share reaches $28.4 \%$, while for $\alpha=20^{\circ}$ it is $35.6 \%$. 


\section{ACKNOWLEDGEMENT}

This work has been financed by the Polish National Centre for Research and Development under the LIDER program Grant Agreement No. LIDER/27/0140/L-10/18/NCBR/2019.

\section{REFERENCES}

[1] Antoniadis, A. F.; Drikakis, D.; Zhong, B.; Barakos, G.; Steijl, R.; Biava, M.; Vigevano, L.; Brocklehurst, A.; Boelens, O.; Dietz, M.; Embacher, M.; Khier, W. (2012). Assessment of CFD methods against experimental flow measurements for helicopter flows, Aerospace Science and Technology, Vol. 19, No. 1, 86-100, doi:10.1016/j.ast.2011.09.003

[2] Beechook, A.; Wang, J. (2013). Aerodynamic analysis of variable cant angle winglets for improved aircraft performance, Proceedings of the $19^{\text {th }}$ International Conference on Automation and Computing, 217-222

[3] Kusyumov, A. N.; Mikhailov, S.; Garipov, A. O.; Nikolaev, E. I.; Barakos, G. N. (2013). CFD simulation of fuselage aerodynamics of the "ANSAT" helicopter prototype, Transaction on Control and Mechanical Systems, Vol. 1, No. 7, 318-324

[4] Nicolosi, F.; Della Vecchia, P.; Ciliberti, D.; Cusati, V. (2014). Development of new preliminary design methodologies for regional turboprop aircraft by CFD analyses, $29^{\text {th }}$ Congress of the International Council of the Aeronautical Sciences, 734-744, doi:10.13140/2.1.5147.0721

[5] Li, H. X.; Li, B.; Choi, J.; Heo, J.; Kim, I. (2016). Analysis of a novel nozzle used for pulse jet filtration using CFD simulation method, International Journal of Simulation Modelling, Vol. 15, No. 2, 262-274, doi:10.2507/IJSIMM15(2)6.334

[6] Shah, G. H.; Cunningham, K.; Foster, J. V.; Fremaux, C. M.; Stewart, E. C.; Wilborn, J. E.; Gato, W.; Pratt, D. W. (2002). Wind-tunnel investigation of commercial transport aircraft aerodynamics at extreme flight conditions, SAE Technical Paper Series, Paper 2002-01-2912, 10 pages, doi: $10.4271 / 2002-01-2912$

[7] Harris, F. D. (2003). An Overview of Autogyros and the McDonnell XV-1 Convertiplane, Contractor Report NASA/CR-2003-212799, Ames Research Center, Mountain View

[8] Wilks, S. UAVenture's AirRails Adds Support for Helicopters, from http://uaventure.com/2019/12/04/uaventures-airrails-adds-support-for-helicopters/, accessed on 22-05-2020

[9] Thomson, D. G.; Houston, S. (2008). Advances in understanding autogyro flight dynamics, Proceedings of the $64^{\text {th }}$ American Helicopter Society Annual Forum, 391-403

[10] Coton, F. N.; Smrcek, L.; Patek, Z. (1998). Aerodynamic characteristics of a gyroplane configuration, Journal of Aircraft, Vol. 35, No. 2, 274-279, doi:10.2514/2.2295

[11] Krzysiak, A. (2017). Wind tunnel tests of quad-rotor autogyro model, Journal of KONES, Vol. 24, No. 1, 231-238, doi:10.5604/01.3001.0010.2819

[12] Czyz, Z.; Stryczniewicz, W. (2018). Investigation of aerodynamic interference in a multirotor by PIV method, Advances in Science and Technology Research Journal, Vol. 12, No. 1, 106-114, doi:10.12913/22998624/86475

[13] Czyz, Z.; Karpinski, P.; Stryczniewicz, W. (2020). Measurement of the flow field generated by multicopter propellers, Sensors, Vol. 20, No. 19, Paper 5537, 22 pages, doi:10.3390/s20195537

[14] Czyz, Z.; Karpinski, P.; Lusiak, T.; Szczepanik, T. (2017). Numerical analysis of the influence of particular autogyro parts on the aerodynamic forces, ITM Web of Conferences, Vol. 15, Paper 07008, 6 pages, doi:10.1051/itmconf/20171507008

[15] Czyz, Z.; Karpinski, P. (2020). Numerical analysis of the impact of sideslip angle on load of the gyrocopter stabilizers, Aviation, Vol. 23, No. 4, 114-122, doi:10.3846/aviation.2019.11924

[16] Czyz, Z.; Lusiak, T.; Karpinski, P.; Czarnigowski, J. (2018). Numerical investigation of the gyroplane longitudinal static stability for the selected stabilizer angles, Journal of Physics: Conference Series, Vol. 1101, Paper 012003, 8 pages, doi:10.1088/1742-6596/1101/1/012003

[17] Houston, S. S. (1996). Longitudinal stability of gyroplanes, The Aeronautical Journal, Vol. 100, No. 991, 1-6, doi:10.1017/S0001924000027196

[18] Houston, S. S. (1998). Identification of autogyro longitudinal stability and control characteristics, Journal of Guidance, Control, and Dynamics, Vol. 21, No. 3, 391-399, doi:10.2514/2.4271 
[19] Houston, S. S. (1998). Identification of gyroplane lateral/directional stability and control characteristics from flight test, Proceedings of the Institution of Mechanical Engineers, Part G: Journal of Aerospace Engineering, Vol. 212, No. 4, 271-285, doi:10.1243/0954410981532432

[20] Wang, J. C.; Li, J. B. (2014). Effects of wing on autogyro longitudinal stability, Acta Aeronautica et Astronautica Sinica, Vol. 35, No. 1, 151-160

[21] Leishman, G. J. (2006). Principles of Helicopter Aerodynamics, $2^{\text {nd }}$ edition, Cambridge University Press, Cambridge

[22] Eglin, P. (1997). Aerodynamic design of the NH90 helicopter stabilizer, Proceedings of the $23^{\text {rd }}$ European Rotorcraft Forum, 68.1-68.10

[23] Minervino, M.; Vitagliano, P. L.; Quagliarella, D. (2016). Helicopter stabilizer optimization considering rotor downwash in forward-flight, Aircraft Engineering and Aerospace Technology, Vol. 88, No. 6, 846-865, doi:10.1108/AEAT-03-2015-0082

[24] Figat, M. (2017). Aerodynamics analysis of the main rotor influence on the static stability of the gyroplane, Aircraft Engineering and Aerospace Technology, Vol. 89, No. 5, 663-670, doi:10.1108/AEAT-01-2017-0047

[25] Batrakov, A.; Garipova, L.; Kusyumov, A.; Mikhailov, S.; Barakos, G. (2015). Computational fluid dynamics modeling of helicopter fuselage drag, Journal of Aircraft, Vol. 52, No. 5, 1634-1643, doi:10.2514/1.C033019

[26] Filippone, A. (2007). Prediction of aerodynamic forces on a helicopter fuselage, The Aeronautical Journal, Vol. 111, No. 1117, 175-184, doi:10.1017/S0001924000004437

[27] Sheikhi, H.; Saghaie, A. (2017). Developing an engineering-statistical model for estimating aerodynamic coefficients of helicopter fuselage, Chinese Journal of Aeronautics, Vol. 30, No. 1, 175-185, doi:10.1016/j.cja.2016.12.015

[28] Jeong, J.; Hussain, F. (1995). On the identification of a vortex, Journal of Fluid Mechanics, Vol. 285, 69-94, doi:10.1017/S0022112095000462

[29] Jiang, M.; Machiraju, R.; Thompson, D. (2002). A novel approach to vortex core region detection, Proceedings of the 2002 Joint EUROGRAPHICS-IEEE TCVG Symposium on Visualisation, 217225

[30] Van Gelder, A. (2012). Vortex core detection: back to basics, Proceedings of the 2012 Visualization and Data Analysis, Vol. 8294, Paper 829413, 8 pages, doi:10.1117/12.912301

[31] Jiang, M.; Machiraju, R.; Thompson, D. (2005). Detection and visualization of vortices, Hansen, C. D.; Johnson, C. R. (Eds.), The Visualization Handbook, Elsevier, Amsterdam, 295-309, doi:10.1016/B978-012387582-2/50016-2

[32] Sujudi, D.; Haimes, R. (1995). Identification of swirling flow in 3-D vector fields, Proceedings of the $12^{\text {th }}$ Computational Fluid Dynamics Conference, 792-799, doi:10.2514/6.1995-1715

[33] Ansys (2010). Ansys 13.0 User's Guide, ANSYS Inc., Canonsburg

[34] Rajan, G. K.; Cimbala, J. M. (2017). Computational and theoretical analyses of the precessing vortex rope in a simplified draft tube of a scaled model of a Francis turbine, Journal of Fluids Engineering, Vol. 139, No. 2, Paper 021102, 12 pages, doi:10.1115/1.4034693

[35] Kenwright, D.; Haimes, R. (1997). Vortex identification-applications in aerodynamics: a case study, Proceedings of the 1997 Visualization, 413-416, doi:10.1109/VISUAL.1997.663910

[36] Kenwright, D. N.; Haimes, R. (1998). Automatic vortex core detection, IEEE Computer Graphics and Applications, Vol. 18, No. 4, 70-74, doi: $10.1109 / 38.689668$ 